\title{
Linkage Mechanism Optimization and Sensitivity Analysis of an Automotive Engine Hood
}

\author{
Onur Denizhan ${ }^{1}$, Meng-Sang Chew ${ }^{1 *}$ \\ ${ }^{1}$ Department of Mechanical Engineering and Mechanics, Lehigh University, Bethlehem, PA, 18015, United States
}

\begin{abstract}
Equipment such as a rod between vehicle body and hood or a lift strut is used to keep an automobile hood in open positions during regular maintenance or repairs. Moreover, some effort is required to open or close the automobile hood due to its weight. To keep it in various opened positions and to exert the least force to open or close it, a mechanism is used in conjunction with extension, compression or torsion springs in two different configurations. Each optimization has been carried out for all these different springs, each in two configurations and results are compared. Sensitivity analysis for the design variables are examined to determine how these variables are influential on the designed mechanism. Optimization results for extension and compression springs are observed to be similar in both configurations of the designed mechanism. In addition to that, optimization results of torsion spring are observed to be similar with extension and compression springs optimization results for second configuration. Overall, the compression spring application gives the best optimal results for both configurations of the designed mechanism. Sensitivity analysis shows that the designed mechanism is not sensitive to design variables that are on the bounds in both of these configurations.
\end{abstract}

Keywords: Automobile engine hood; Linkage mechanism; Mechanism synthesis; Nonlinear optimization; Sensitivity analysis; Springs.

\author{
* Corresponding author \\ Meng-Sang Chew \\ mc0p@lehigh.edu
}

Address: Packard Lab. 256B

19 Memorial Dr. West, Bethlehem, PA, 18015, United States

Tel: +16107584215

Fax: +16107586224

Manuscript Received 11.12.2017

Revised 27.02.2018

Accepted 27.02.2018

Doi:10.30939/ijastech..364438

\section{Introduction}

Vehicles need to have regular maintenance and repair so that hoods need to be readily opened and closed. This requires that automobile hoods have lower opening and closing efforts. On the other hand, automotive engine hoods may be too heavy for some and a mechanism maybe needed to assist in opening and keeping the hood in an open position. Previously, gas lifters were installed to lift vehicle engine hood and lid and detail explanation on the mathematical modeling of the moving mechanism was investigated $[1,2]$.

In this article, a linkage mechanism was designed that includes a spring for storing potential energy during the hood closing and released on opening. What is required and needed in the design is to locate the attachment points of the spring on the linkage of mechanism to keep the hood in a range of stable opened positions. In addition to that sensitivity analysis was investigated for design variables that are on the bounds.

\section{Designed Mechanism}

There are many kinds of mechanisms for automotive engine hoods that can keep engine hoods at different open positions. Along with that, there are components such as springs, dampers or props. In this study, a linkage mechanism was designed and is shown Figure 1. Three kinds of spring are used: extension, compression and torsion spring, each applied to the first link or second link. The basic goal is to exert a minimum force for opening or closing the engine hood.

Table 1. Designed Link Lengths

\begin{tabular}{c|c}
\hline Link & Link Length $(\mathrm{m})$. \\
\hline Link $[\mathrm{AC}]\left(l_{2}\right)$ & 0.40 \\
\hline Link [CD] $\left(l_{3}\right)$ & 0.15 \\
\hline Link [DE] $\left(l_{4}\right)$ & 0.35 \\
\hline Link [CP] $\left(l_{p}\right)$ & 1 \\
\hline
\end{tabular}


In this article, the design synthesis of the hood linkage [3] has been omitted. This study concentrates on determining the various spring parameters optimally to statically balance the hood.

In the designed linkage mechanism, the hood weight is assumed to $12 \mathrm{~kg}$. a representative of the weight of automobile engine hoods and angle $\alpha$ shown in Figure 2, is set at 16 degrees. Link lengths are given in Table 1 for the designed linkage mechanism.

Table 2. Link Angles for Open and Close Positions

\begin{tabular}{c|c|c|c}
\hline $\begin{array}{c}\text { Hood } \\
\text { Position }\end{array}$ & $\begin{array}{c}\text { Link }[\mathrm{AC}] \\
\text { (deg.) }\end{array}$ & $\begin{array}{c}\text { Link }[\mathrm{CD}] \\
\text { (deg.) }\end{array}$ & $\begin{array}{c}\text { Link [DE] } \\
\text { (deg.) }\end{array}$ \\
\hline Open & 60 & 38.6 & 103.7 \\
\hline Close & 89.2 & 25.5 & 137.7 \\
\hline
\end{tabular}

In addition to that angles of the links of the designed mechanism for open and close positions are shown in Table 2 above. Moreover, the hood angle in the closed and opened positions are shown in Table 3 below so that the hood angle is 54.6 degrees when fully opened and -9.5 degrees at the fully close position, thereby providing a good range of motion for repair or maintenance.

Table 3. Hood Angle for Open and Close Positions

\begin{tabular}{c|c}
\hline Hood Position & $\begin{array}{c}\text { Angle of the Hood } \\
\text { (deg.) }\end{array}$ \\
\hline Open & 54.6 \\
\hline Close & -9.5 \\
\hline
\end{tabular}

The hood is closing when first and second links rotated counter-clockwise and the hood is opening when they rotate clockwise.

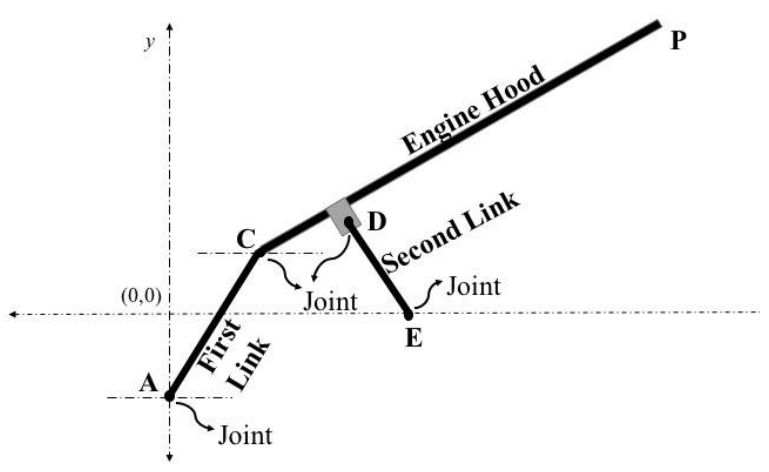

Figure 1. Designed Engine Hood Mechanism

\section{Extension Spring Application}

A helical extension spring absorbs and stores energy by offering resistance to a pulling force. Various types of ends are used to attach this type of spring to the source of the force. Literature on numerical methods provides several examples of extension spring optimization. Different researchers used springs to illustrate different optimization methods with mixed variables in some articles $[4,5,6]$, and they are applied to oversimplified mathematical model. Parades et. al [7] performed an optimization using an industrial software package, with given specifications for extension springs. Another example used extension springs for static balancing of weights in a given mechanical system that operates in a vertical plane [8]. All of these studies show that helical extension springs have been applied to all kinds of engineering applications.

\subsection{First Link Application (Extension Spring)}

The linkage shown in Figure 2 consists of an extension spring that is attached to first link [AC] of linkage. During the operation of this hood linkage, the extension spring stores potential energy of the hood that is given up with hood closing, and the stored energy is released when the hood opens. During optimization, $\boldsymbol{k}_{\boldsymbol{e}}, \boldsymbol{\beta}, \boldsymbol{l}_{\mathbf{0}_{e}}, \boldsymbol{b}, \boldsymbol{x}_{\boldsymbol{s}}$ and $\boldsymbol{y}_{\boldsymbol{s}}$ are specified as design variables that are defined in nomenclature part in end of this article and shown in Figure 2 below for an extension spring, attached to the first link $[\mathrm{AC}]$ at point $\mathrm{B}$.

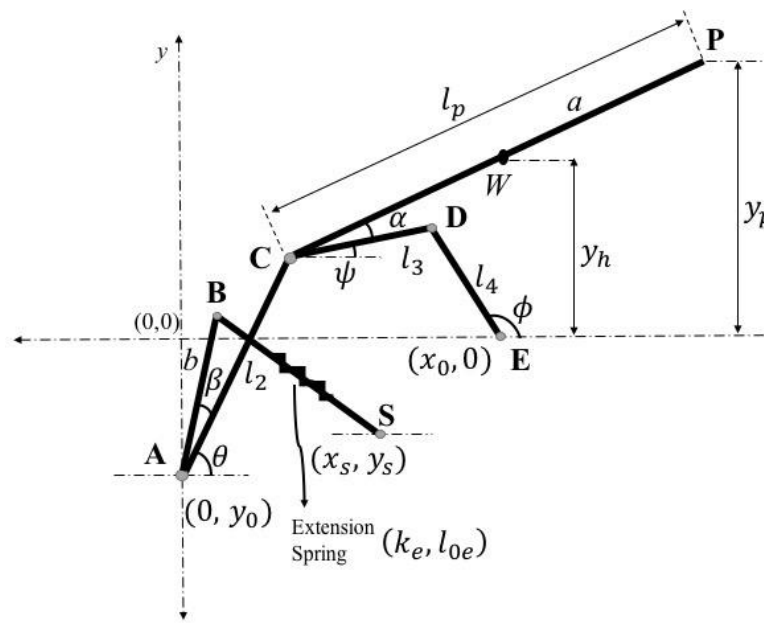

Figure 2. Extension Spring First Link Application

\subsubsection{Problem Formulization}

Loop closure equation is below:

$$
l_{2} e^{i \theta}+l_{3} e^{i \psi}-l_{4} e^{i \phi}-x_{0}-i y_{0}=0
$$

where angle $\boldsymbol{\theta}$ is the position of the first link. 
Velocity coefficients of the mechanism:

$$
\begin{gathered}
-l_{2} \sin \theta \delta \theta-\sin \psi F_{\psi} \delta \theta+l_{4} \sin \phi F_{\phi} \delta \theta=0 \\
l_{2} \cos \theta \delta \theta+l_{3} \cos \psi F_{\psi} \delta \theta-l_{4} \cos \phi F_{\phi} \delta \theta \\
=0
\end{gathered}
$$

Eq. (2) and Eq. (3) were solved for $\boldsymbol{F}_{\boldsymbol{\psi}}$ and $\boldsymbol{F}_{\boldsymbol{\phi}}$.

Length of spring:

$$
\begin{aligned}
& l_{s}^{2}=\left(b_{x}-x_{s}\right)^{2}+\left(b_{y}-y_{s}\right)^{2} \\
& b_{x}=b \cos (\theta+\beta) \\
& b_{y}=b \sin (\theta+\beta)
\end{aligned}
$$

Position of center of the hood for $y$ axis:

$$
y_{h}=-y_{0}+l_{2} \sin \theta+a \sin (\psi+\alpha)
$$

Position of the $\mathrm{P}$ point for $y$ axis:

$$
y_{p}=-y_{0}+l_{2} \sin \theta+l_{p} \sin (\psi+\alpha)
$$

Virtual displacements:

$$
\begin{gathered}
\delta l_{s}=\frac{-b\left(b_{x}-x_{s}\right) \sin (\theta+\beta)}{l_{s}} \delta \theta+ \\
\frac{b\left(b_{y}-y_{s}\right) \cos (\theta+\beta)}{l_{s}} \delta \theta \\
\delta y_{h}=l_{2} \cos \theta \delta \theta+a \cos (\psi+\alpha) F_{\psi} \delta \theta \\
\delta y_{p}=l_{2} \cos \theta \delta \theta+l_{p} \cos (\psi+\alpha) F_{\psi} \delta \theta
\end{gathered}
$$

Conservative potentials:

(i) For hood: $\quad \boldsymbol{\delta} \boldsymbol{V}_{\boldsymbol{h}}=\boldsymbol{W} \boldsymbol{\delta} \boldsymbol{y}_{\boldsymbol{h}}$

(ii)For spring: $\quad \boldsymbol{\delta} \boldsymbol{V}_{s}=\boldsymbol{k}_{e}\left[\boldsymbol{l}_{\boldsymbol{s}}-\boldsymbol{l}_{\mathbf{0} e}\right] \boldsymbol{\delta} \boldsymbol{l}_{\boldsymbol{s}}$

Virtual work:

$$
\delta W=P_{a} \delta y_{p}-\delta V_{s}-\delta V_{h}
$$

so that the upward force on the hood is:

$$
\begin{aligned}
P_{a}= & \frac{W\left[l_{2} \cos \theta+a \cos (\psi+\alpha) F_{\psi}\right]}{d_{1}}+ \\
& \frac{-k_{e}\left(l_{s}-l_{0 e}\right) \frac{b}{l_{s}}\left(b_{x}-x_{s}\right) \sin (\theta+\beta)}{d_{1}}+ \\
& \frac{k_{e}\left(l_{s}-l_{0 e}\right) \frac{b}{l_{s}}\left(b_{y}-y_{s}\right) \cos (\theta+\beta)}{d_{1}}
\end{aligned}
$$

$$
\text { where } \quad d_{1}=l_{2} \cos \theta+l_{p} \cos (\psi+\alpha) F_{\psi}
$$

and $\boldsymbol{P}_{\boldsymbol{a}}$ is the vertical force need by a person to open or close the hood.

\subsection{Second Link Application (Extension Spring)}

The configuration is shown in Figure 3 consists of an extension spring that is attached to second link [DE] of linkage. During optimization, $\boldsymbol{k}_{\boldsymbol{e}}, \boldsymbol{\beta}, \boldsymbol{l}_{\mathbf{0} \boldsymbol{e}}, \boldsymbol{b}, \boldsymbol{x}_{\boldsymbol{s}}$ and $\boldsymbol{y}_{\boldsymbol{s}}$ are specified as design variables. These are all shown in Figure 3 for an extension spring. Note that in this case the angle $\boldsymbol{\theta}$ is the position of the second link. Equations of the extension spring second link configuration is shown in Appendix $\mathrm{A}$ in end of this article.

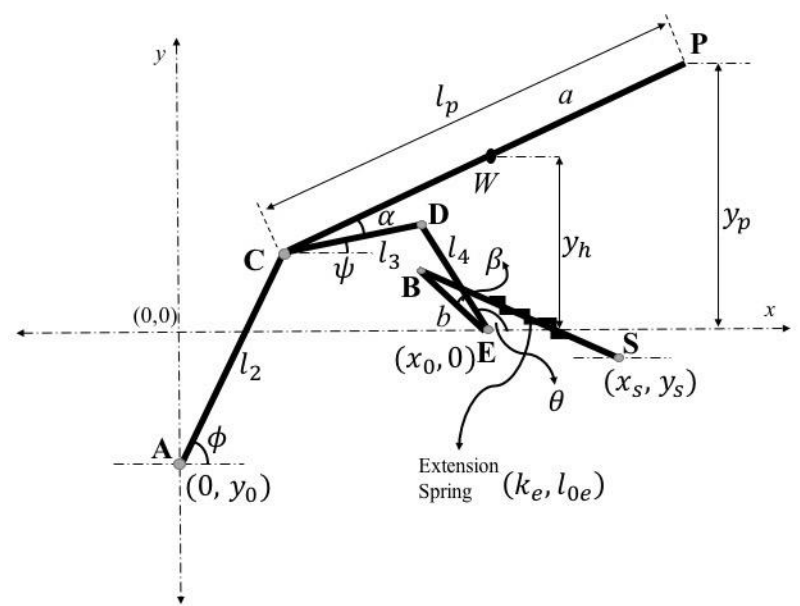

Figure 3. Extension Spring Second Link Application

\section{Compression Spring Application}

Most of patents and articles focuses on tension and material of compression springs today. One example of a prior compression spring patent shows that compression springs are used for vehicle suspension systems in U.S. Pat. No. 3,892,398 to Marsh, et al [9]. One of the another article by Genova [10] provides general and important points in planar mechanisms with springs.

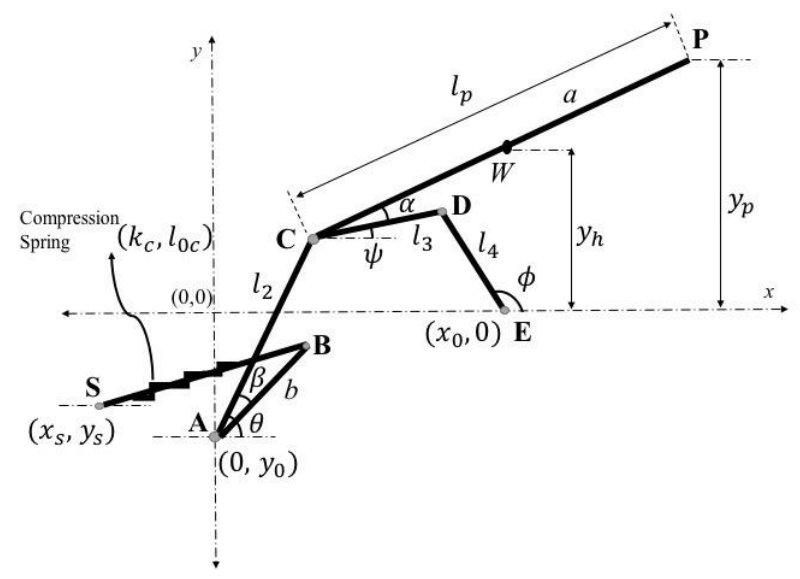

Figure 4. Compression Spring First Link Application 
In addition to that Matthew and Tesar [11] showed independent parametric relationships with links contiguous or noncontiguous with the links which have springs. Furthermore, Kurt Hain [12] created force characteristics in general form for different spring mechanisms which include helical compression springs.

\subsection{First Link Application (Compression Spring)}

This configuration is shown in Figure 4. The compression spring is attached to first link [AC] of linkage. During optimization, $\boldsymbol{k}_{\boldsymbol{c}}, \boldsymbol{\beta}, \boldsymbol{l}_{\mathbf{0} c}, \boldsymbol{b}, \boldsymbol{x}_{\boldsymbol{s}}$ and $\boldsymbol{y}_{\boldsymbol{s}}$ are specified as design variables. All of these are shown Figure 4 for a compression spring. Appendix B includes equations of this case.

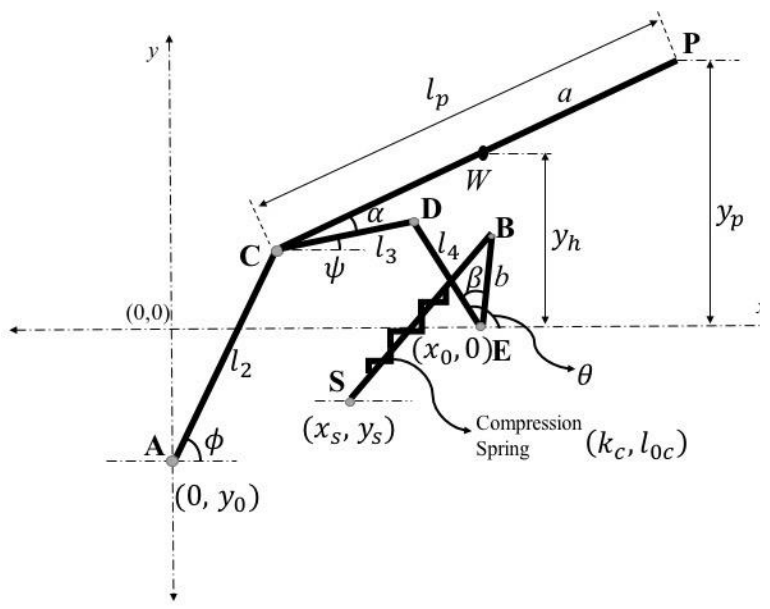

Figure 5. Compression Spring Second Link Application

\subsection{Second Link Application (Compression Spring)}

The hood configuration is shown in Figure 5 above. A compression spring is then applied to second link [DE] of the system. During operation of hood, the compression spring stores potential energy, when the hood is closed and

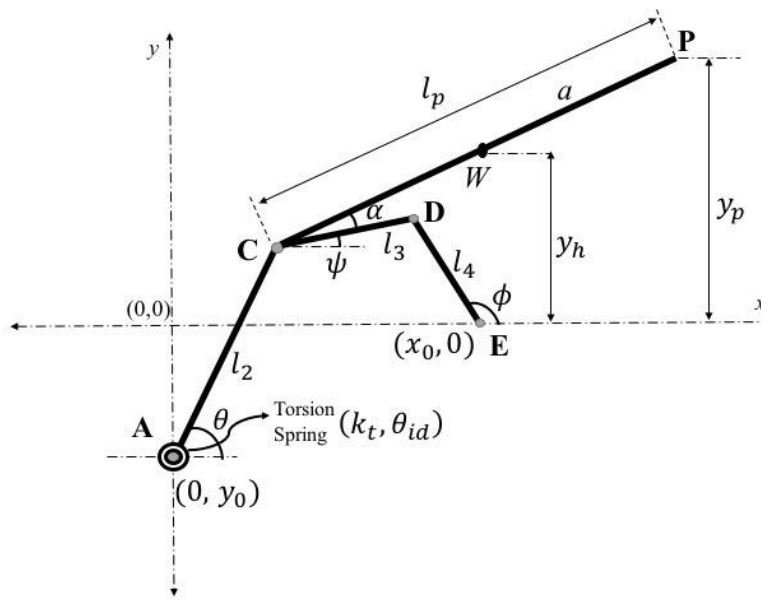

Figure 6. Torsion Spring First Link Application

the spring releases back the store an energy when the hood is opened. During optimization, $\boldsymbol{k}_{\boldsymbol{c}}, \boldsymbol{\beta}, \boldsymbol{l}_{\mathbf{0} \boldsymbol{c}}, \boldsymbol{b}, \boldsymbol{x}_{\boldsymbol{s}}$ and $\boldsymbol{y}_{\boldsymbol{s}}$ are taken the design variables as same with first link application of compression spring. These are shown Figure 5 for a compression spring. Equation of the designed mechanism for this configuration is shown in Appendix C.

\section{Torsion Spring Application}

A torsion spring is a spring that works by torsion or twisting; that is, a flexible elastic object that stores mechanical energy when it is twisted. When it is twisted, it exerts a torque in the opposite direction and proportional to the angle it is twisted. Shigley, et al. [13] gives details about torsion spring working principles such as those mentioned above. The torsion spring configuration is created for the purpose of storing and releasing angular energy or for the purpose of statically holding a mechanism in place by deflecting the legs about the body centerline axis. There are different kinds of application for torsion spring; one example is a torsion spring that is normally supported by a rod (mandrel) that is coincident with the theoretical hinge line of the final product [14].

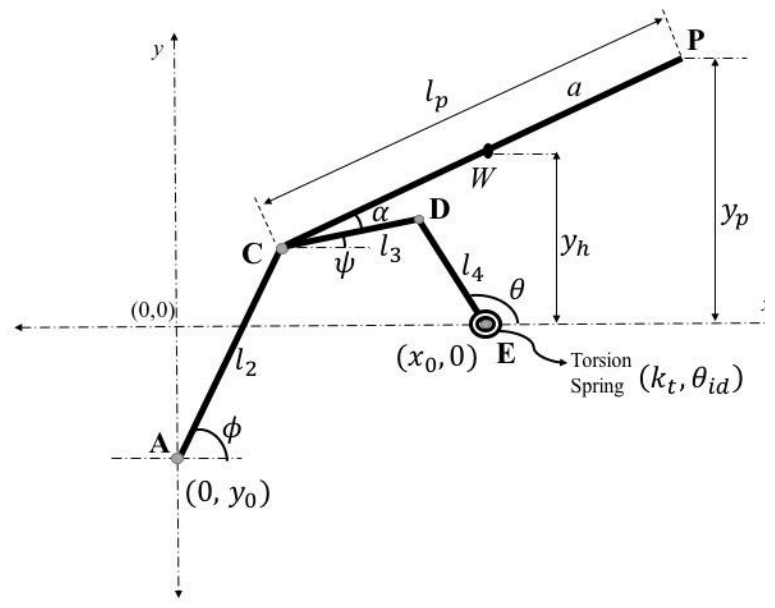

Figure 7. Torsion Spring Second Link Application

There are also a number of torsion spring balance assembly patents and different applications. One example of a prior spring balance assembly is shown in U.S. Pat. No. 3,038,714 by Klaus, et al [15]. The spring tension can therefore be adjusted (e.g., increased or decreased) by either counter-clockwise or clockwise rotational movement of a spring regulator and this patent design allows the coil spring to be either tightened or loosened to the degree necessary to sufficiently support or position a loading arm. Another prior spring assembly unit, for 
example, as seen in U.S. Pat. No. 4,537,233 by Vroonland, et al [16], which had a protectively covered spring torsion unit with an assembly of a threaded adjustment screw and barrel nut. Both of these patents show how torsion springs can be adjusted in different ways.

All of these applications show that torsion springs are used in mechanical systems for static balancing or dynamic balancing in a number of application fields.

\subsection{First Link Application (Torsion Spring)}

In the designed linkage mechanism for this case, as shown in Figure 6, the torsion spring is applied to the pivot point of the first link [AC] of the mechanism. During hood motion, the torsion spring stores mechanical energy that is given up by the closing of the hood and releases it back when the hood is open. In this configuration, note that the second link [DE] is left without any attachment to any other mechanical assistance. During optimization, torsion spring constant $\boldsymbol{k}_{\boldsymbol{t}}$ and initial angular deflection of the torsion spring $\boldsymbol{\theta}_{\boldsymbol{i}}$ were taken as design variables. Equations for torsion spring first link application are shown in Appendix D.

\subsection{Second Link Application (Torsion Spring)}

The linkage system for the case is shown in Figure 7. In this situation, the torsion spring is applied to the pivot point of second link [DE] of system. During the torsion spring application, the torsion spring stores mechanical energy that is given up by the closing of the hood and releases it back when the hood is open. In this configuration, the first link $[\mathrm{AC}]$ is left without any attachment to any other mechanical assistance. During optimization, $\boldsymbol{k}_{\boldsymbol{t}}, \boldsymbol{\theta}_{\boldsymbol{i d}}$ were taken as design variables. Designed mechanism equations for this case are shown in Appendix E.

\section{Results}

There are six design variables each configuration of the extension and compression springs and there two design variables for each torsion spring application. During the optimization process, designed upper and lower boundary constraints for the design variables are shown in Tables 4 and 5 below.

Table 4. Boundary Conditions of Torsion Spring

\begin{tabular}{c|c|c|c}
\hline \multicolumn{2}{c|}{} & First Link & Second Link \\
\hline \multirow{2}{*}{\begin{tabular}{c} 
(N.m/radian) \\
\cline { 2 - 4 }
\end{tabular}} & Upper & 20000 & 20000 \\
\cline { 2 - 4 }$\theta_{\text {id }}$ & Lower & 0.1 & 0.1 \\
\cline { 2 - 4 }$($ radian $)$ & Lower & 0.873 & 0.873 \\
\hline
\end{tabular}

In Table 8, sensitivity analysis results are shown. Sensitivity analysis only was carried out for design variables that that are on the bound. These variables are $x$ for extension spring first and second link configurations and compression spring second link application, variable $y$ for extension spring second link application and variable $b$ for second link compression spring application. During the sensitivity analysis process, only these variables boundary conditions were relaxed and all other boundary conditions of the design variables were not changed.

Table 5. Boundary Conditions of Extension and Compression Springs

\begin{tabular}{|c|c|c|c|c|c|}
\hline & & \multicolumn{2}{|c|}{ Extension Spring } & \multicolumn{2}{|c|}{$\begin{array}{c}\text { Compression } \\
\text { Spring }\end{array}$} \\
\hline & & $\begin{array}{l}\text { First } \\
\text { Link }\end{array}$ & $\begin{array}{l}\text { Second } \\
\text { Link }\end{array}$ & $\begin{array}{l}\text { First } \\
\text { Link }\end{array}$ & $\begin{array}{l}\text { Second } \\
\text { Link }\end{array}$ \\
\hline \multirow{2}{*}{$\begin{array}{c}k \\
(\mathrm{~N} / \mathrm{m})\end{array}$} & Upper & $10^{6}$ & $10^{6}$ & $10^{6}$ & $10^{6}$ \\
\hline & Lower & 0.1 & 0.1 & 0.1 & 0.1 \\
\hline \multirow{2}{*}{$\begin{array}{c}\beta \\
\text { (deg.) }\end{array}$} & Upper & 359 & 359 & 359 & 359 \\
\hline & Lower & -359 & -359 & -359 & -359 \\
\hline \multirow{2}{*}{$b(\mathrm{~m})$} & Upper & 0.5 & 0.5 & 0.5 & 0.22 \\
\hline & Lower & 0.01 & 0.01 & 0.01 & 0.01 \\
\hline \multirow{2}{*}{$l_{0}(\mathrm{~m})$} & Upper & 0.9 & 0.9 & 0.65 & 0.9 \\
\hline & Lower & 0.001 & 0.001 & 0.001 & 0.001 \\
\hline \multirow{2}{*}{$x(\mathrm{~m})$} & Upper & 0.7 & 0.7 & 0.7 & 0.7 \\
\hline & Lower & -0.5 & -0.5 & -0.5 & -0.5 \\
\hline \multirow{2}{*}{$y(\mathrm{~m})$} & Upper & 0 & 0 & 0 & 0 \\
\hline & Lower & -0.4 & -0.4 & -0.4 & -0.4 \\
\hline
\end{tabular}

Vehicle engine compartment dimensions are main criteria that drives boundary constraints because the linkage mechanism was designed based on practical considerations. On the other hand, vehicle dimensions were not considered when boundary constraints were relaxed for the sensitivity analysis.

Table 6. Optimization Results of Extension and Compression Springs

\begin{tabular}{c|c|c|c|c}
\hline \multirow{2}{*}{} & \multicolumn{2}{|c|}{ Extension Spring } & \multicolumn{2}{c}{ Compression Spring } \\
\cline { 2 - 5 } & $\begin{array}{c}\text { First } \\
\text { Link }\end{array}$ & $\begin{array}{c}\text { Second } \\
\text { Link }\end{array}$ & $\begin{array}{c}\text { First } \\
\text { Link }\end{array}$ & $\begin{array}{c}\text { Second } \\
\text { Link }\end{array}$ \\
\hline$k(\mathrm{~N} / \mathrm{m})$ & 312013 & 211092 & 26028 & 36400 \\
\hline$\beta$ (radian) & -1.163 & -2.423 & -0.473 & -1.437 \\
\hline $\boldsymbol{b}(\mathrm{m})$ & 0.046 & 0.049 & 0.3 & 0.22 \\
\hline$l_{0}(\mathrm{~m})$ & 0.708 & 0.441 & 0.622 & 0.464 \\
\hline$x(\mathrm{~m})$ & 0.7 & 0.7 & -0.29 & 0.178 \\
\hline$y(\mathrm{~m})$ & -0.395 & -0.4 & -0.177 & 0 \\
\hline
\end{tabular}


applications results for first and second link. In addition to

Table 8. Sensitivity Analysis Results after Optimization

\begin{tabular}{c|c|c|c|c|c}
\hline & $\begin{array}{c}\text { Boundary } \\
\text { Conditions }\end{array}$ & $\begin{array}{c}\text { Objective } \\
\text { Function }\end{array}$ & $\begin{array}{c}\text { Relaxed } \\
\text { Boundary Conditions }\end{array}$ & $\begin{array}{c}\text { Objective Function with } \\
\text { Relaxed Boundary Conditions }\end{array}$ & $\begin{array}{c}\text { Percentage } \\
\text { Change }\end{array}$ \\
\hline $\begin{array}{c}\text { First Link Extension } \\
\text { Spring }\end{array}$ & $\begin{array}{c}-0.5<x<0.7 \\
0.7\end{array}$ & 7722.138 & $\begin{array}{c}-0.5<x<2 \\
0.904\end{array}$ & 7687.354 & -0.45 \\
\hline $\begin{array}{c}\text { Second Link } \\
\text { Extension Spring }\end{array}$ & $\begin{array}{c}-0.5<x<0.7 \\
0.7\end{array}$ & 3255.331 & $\begin{array}{c}-0.5<x<2 \\
1.29\end{array}$ & 3157.985 & -2.99 \\
\hline Second Link & $\begin{array}{c}-0.4<y<0 \\
-0.4\end{array}$ & 3255.331 & $\begin{array}{c}-2<y<0 \\
-0.912\end{array}$ & 3157.988 & -2.99 \\
\hline Extension Spring & $\begin{array}{c}0.22 \\
\text { Second Link } \\
\text { Compression Spring }\end{array}$ & $\begin{array}{c}0.01<0.22 \\
0<0<2\end{array}$ & 2556.838 & 0 \\
\hline $\begin{array}{c}\text { Second Link } \\
\text { Compression Spring }\end{array}$ & $\begin{array}{c}-0.4<x<0 \\
0\end{array}$ & 2556.854 & $\begin{array}{c}0.01<y<2 \\
0.01\end{array}$ & 2556.854 & 0 \\
\hline
\end{tabular}

The optimization results are shown in Tables 6 and 7. For torsion spring applications, first and second link configurations optimum values are different in Table 7 but optimum objective function values are the same. In optimization process, while zero-spring length of extension spring is smaller than extension spring length during the hood motion, compression spring zero-spring length should be larger than compression spring length during the motion because of these springs working principles.

Table 7. Optimization Results of Torsion Springs

\begin{tabular}{c|c|c}
\hline & $\begin{array}{c}k_{t} \\
\text { (N.m/radian) }\end{array}$ & $\begin{array}{c}\theta_{\text {id }} \\
\text { (radian) }\end{array}$ \\
\hline First Link & 280.968 & 0.112 \\
\hline Second Link & 241.302 & 0.13 \\
\hline
\end{tabular}

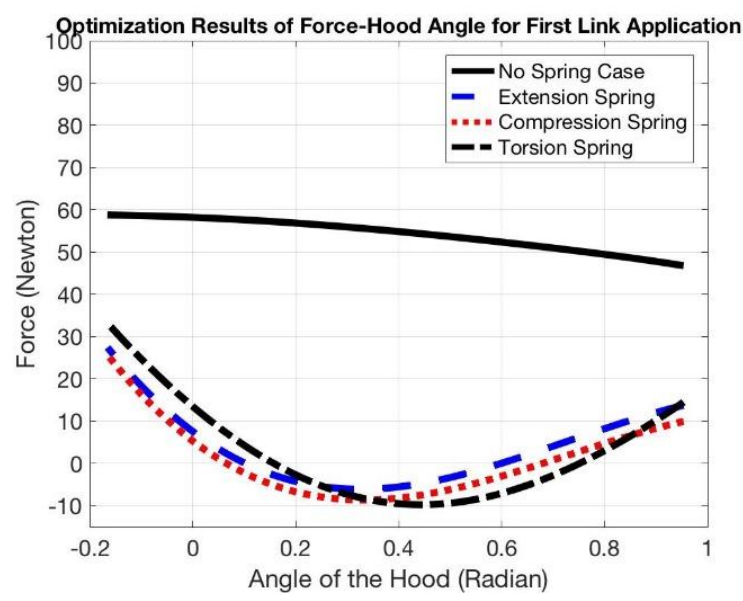

Figure 8. Optimization results for first link

Figures 8 and 9 are shown force - hood angle graphs for extension, compression and torsion spring that force requirements are shown in these figures if there is not any spring on the mechanism and called as "No Spring Case".

\section{Discussion and Conclusion}

Note that the optimum values for $x, y$ and $b$ (on the second column of Table 8) are at the bounds. A relaxation of the bounds (fourth column of Table 8) results in only a slightly lower objective function value, even though the new optimum values are no longer at the bound. This means that the optimum mechanism is not very sensitive to changes in the bounds for variables $x$ and $y$. As shown, the objective function improvement is less then $3 \%$ and that percentage change is small relaxation to the large relaxation in the boundary.

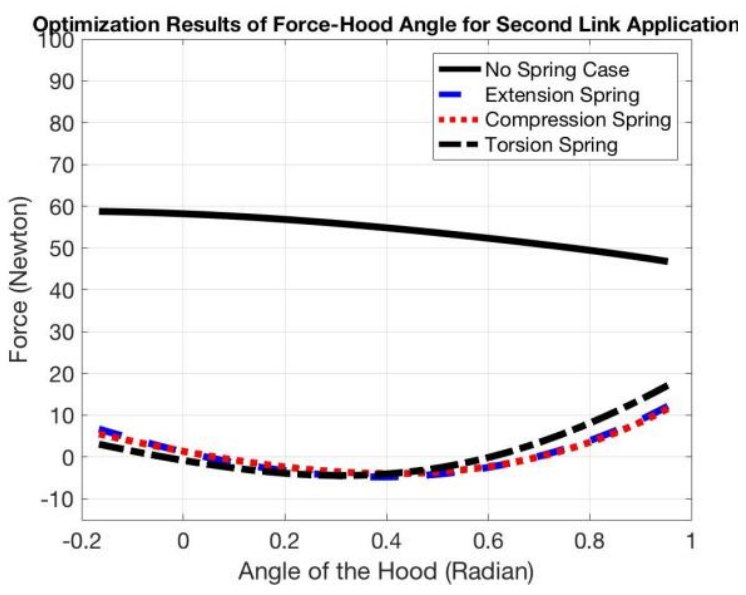

Figure 9. Optimization results for second link

Figures 8 and 9 show that the designed mechanism in the presence of spring balancing reduces the upward force required to raise the hood. In Figure 8, the springs are applied to first link, while in Figure 9, they are applied to 
second link. Moreover, "No Spring Case" curve shows that designed mechanism slightly reduces force requirement without any spring application.

Figures 8 and 9 also show that the compression spring is as optimal when compared with results of extension and torsion spring because the force requirements to raise the hood with a compression spring are smaller than for extension and torsion springs. In addition to that, when the extension, compression and torsion spring results are compared, the second link configuration with a compression spring is the best solution for the designed mechanism. This is because the results of compression spring for second link configuration show that the magnitude of the overall force for opening the hood is the smallest in all of the cases.

\section{Acknowledgment}

Authors wish to express their gratitude to the Ministry of National Education of Republic of Turkey which indirectly made this work possible.

\section{Nomenclature}

$\alpha \quad:$ Angle between links [PC] and [CD] (degree)

$\beta \quad:$ Angle between links $[\mathrm{AB}]$ and $[\mathrm{AC}]$ (degree)

$\phi \quad$ : Passive link angle (degree)

$\psi \quad$ : Angle of link [CD] (degree)

$\theta \quad:$ Active link angle (degree)

$\theta_{d} \quad:$ Deflection angle of torsion spring during the moti on (degree)

$\theta_{i d} \quad$ : Angle of initial deflection from rest position of tor sion spring (degree)

$b \quad$ : Length of link $[\mathrm{AB}]$

$b_{x} b_{y} \quad$ : Point B coordinates

$F_{\phi} F_{\psi} \quad$ : Velocity coefficients of the system

$k_{c} \quad$ : Compression spring constant

$k_{e} \quad:$ : Extension spring constant

$k_{t} \quad$ : Torsion spring constant

$l_{2} \quad:$ Length of link [AC]

$l_{3} \quad:$ Length of link [CD]

$l_{4} \quad:$ Length of link [DE]

$l_{p} \quad:$ Length of link [CP]

$l_{s} \quad:$ Length of spring during the motion

$l_{0 c} \quad:$ Zero-length of compression spring

loe : Zero-length of extension spring

W : Work

$x_{s}, y_{s} \quad:$ Spring attachment point coordinates

$y_{h} \quad$ : Center of mass point of the hood $\mathrm{Y}$ axis coordinates

$y_{p} \quad$ : Applied force $\mathrm{Y}$ axis coordinate

\section{References}

[1] Choi, K.S. et al. (2007). Dynamic analysis of vehicle trunk lid with 4-bar link structure. World Congress, Detroit, Michigan, April 16-19.

[2] Ko, O.S. (1995). The mechanism analysis on hood, tail gate, and trunk lid by mathematical modeling. International Congress and Exposition, Detroit, Michigan, February 27 March 2.

[3] Norton, R.L. (1999). Design of machinery. McGraw-Hill

[4] Deb, K. and Goyal, M. (1998). Flexible optimization procedure for mechanical component design based on genetic adaptive search. Journal of Mechanical Design 120 (2): 162164.

[5] Kannan, B.K. and Kramer, S.N. (1994). An augmented lagrange multiplier based method for mixed integer discrete continuous optimization and its applications to mechanical design. Journal of Mechanical Design 116 (2): 405-411.

[6] Sandgren, E. (1990). Nonlinear integer and discrete programming in mechanical design optimization. Journal of Mechanical Design 112: 223-229.

[7] Parades, M. et al. (2001). An optimization process for extension spring design. Computer Methods in Applied Mechanics and Engineering, 191 (8-10): 783-797.

[8] Nathan, R.H. (1985). A constant force generation mechanism. Journal of Mechanisms, Transmissions and Automation in Design, 107: 508-512.

[9] Marsh, G.L. (1975). Compression spring. United States: Patent 3,892,398.

[10]Genova, P.E. (1972). Mechanics of plane mechanisms with spring links. VI Lenin, Sofia, Bulgaria: Mechanical and Electro-Technical Institute.

[11]Matthew, G.K. and Tezar, D. (1977). Synthesis of spring parameters to balance general forcing functions in planar mechanism. Journal of Engineering for Industry, 347-357.

[12]Hain, K. (1961). Spring mechanisms point balancing. Spring Design and Application, New York, McGraw-Hill, 268-275.

[13]Shigley, J.E. et al. (2003). Mechanical engineering design. McGraw, New York, 542.

[14]Century Springs Company (2003). Torsion springs. Engineers Guide.

[15]Schwieterman, M.A. and Hutchinson, D.E. (1996). Torsion spring balance assembly and adjustment method. United States: Patent 5,893,936.

[16] Vrooland, E. J. and Curtis, M. (1985). Spring balance assembly. United States: Patent 4,537,233.

\section{Appendix}

\section{Appendix A. Second Link Extension Spring}

Loop closure equations is below:

$$
l_{2} e^{i \phi}+l_{3} e^{i \psi}-l_{4} e^{i \theta}-x_{0}-i y_{0}=0
$$

where angle $\boldsymbol{\theta}$ is the position of the second link.

Velocity coefficients of the system: 
$l_{2} \cos \phi F_{\phi} \delta \theta+l_{3} \cos \psi F_{\psi} \delta \theta-l_{4} \cos \theta \delta \theta$ $=\mathbf{0}$

solved for $\boldsymbol{F}_{\boldsymbol{\psi}}$ and $\boldsymbol{F}_{\boldsymbol{\phi}}$.

Length of springs:

$$
\begin{aligned}
& l_{s}^{2}=\left(b_{x}-x_{s}\right)^{2}+\left(b_{y}-y_{s}\right)^{2} \\
& b_{x}=b \cos (\theta+\beta) \\
& b_{y}=b \sin (\theta+\beta)
\end{aligned}
$$

Position of center of the hood for $y$ axis:

$$
y_{h}=-y_{0}+l_{2} \sin \phi+a \sin (\psi+\alpha)
$$

Position of the $\mathrm{P}$ point for $y$ axis:

$$
y_{p}=-y_{0}+l_{2} \sin \phi+l_{p} \sin (\psi+\alpha)
$$

Virtual displacements:

$$
\begin{gathered}
\delta l_{s}=\frac{-b\left(b_{x}-x_{s}\right) \sin (\theta+\beta)}{l_{s}} \delta \theta+ \\
\frac{b\left(b_{y}-y_{s}\right) \cos (\theta+\beta)}{l_{s}} \delta \theta \\
\delta y_{h}=l_{2} \cos \phi F_{\phi} \delta \theta+a \cos (\psi+\alpha) F_{\psi} \delta \theta \\
\delta y_{p}=l_{2} \cos \phi F_{\phi} \delta \theta+l_{p} \cos (\psi+\alpha) F_{\psi} \delta \theta
\end{gathered}
$$

Conservative potentials:

(i) For hood: $\quad \boldsymbol{\delta} \boldsymbol{V}_{\boldsymbol{h}}=\boldsymbol{W} \boldsymbol{\delta} \boldsymbol{y}_{\boldsymbol{h}}$

(ii) For spring: $\quad \boldsymbol{\delta} \boldsymbol{V}_{\boldsymbol{s}}=\boldsymbol{k}_{\boldsymbol{e}}\left[\boldsymbol{l}_{\boldsymbol{s}}-\boldsymbol{l}_{\boldsymbol{0} e}\right] \boldsymbol{\delta} \boldsymbol{l}_{\boldsymbol{s}}$

Virtual work:

$$
\begin{aligned}
\delta W & =P_{a} \delta y_{p}-\delta V_{s}-\delta V_{h} \\
& =0
\end{aligned}
$$

Force:

$$
P_{a}=\frac{\delta V_{h}+\delta V_{s}}{\delta y_{p}}
$$

where $\boldsymbol{P}_{\boldsymbol{a}}$ is the vertical force need by a person to open the hood.

\section{Appendix B. First Link Compression Spring}

Loop closure equations is below:

$$
l_{2} e^{i \theta}+l_{3} e^{i \psi}-l_{4} e^{i \phi}-x_{0}-i y_{0}=0
$$

where angle $\boldsymbol{\theta}$ is the position of the first link.

Velocity coefficients of the system:

$$
\begin{gathered}
-l_{2} \sin \theta \delta \theta-l_{3} \sin \psi F_{\psi} \delta \theta+l_{4} \sin \phi F_{\phi} \delta \theta \\
=0
\end{gathered}
$$

$l_{2} \cos \theta \delta \theta+l_{3} \cos \psi F_{\psi} \delta \theta-l_{4} \cos \phi F_{\phi} \delta \theta=0$

solved for $\boldsymbol{F}_{\boldsymbol{\psi}}$ and $\boldsymbol{F}_{\boldsymbol{\phi}}$.

Length of springs:

$$
\begin{aligned}
& l_{s}^{2}=\left(b_{x}-x_{s}\right)^{2}+\left(b_{y}-y_{s}\right)^{2} \\
& b_{x}=b \cos (\theta+\beta) \\
& b_{y}=b \sin (\theta+\beta)
\end{aligned}
$$

Position of center of the hood for $y$ axis:

$$
y_{h}=-y_{0}+l_{2} \sin \theta+a \sin (\psi+\alpha)
$$

Position of the $\mathrm{P}$ point for $y$ axis:

$$
y_{p}=-y_{0}+l_{2} \sin \theta+l_{p} \sin (\psi+\alpha)
$$

Virtual displacements:

$$
\begin{gathered}
\delta l_{s}=\frac{-b\left(b_{x}-x_{s}\right) \sin (\theta+\beta)}{l_{s}} \delta \theta+ \\
\frac{b\left(b_{y}-y_{s}\right) \cos (\theta+\beta)}{l_{s}} \delta \theta \\
\delta y_{h}=l_{2} \cos \theta \delta \theta+a \cos (\psi+\alpha) F_{\psi} \delta \theta \\
\delta y_{p}=l_{2} \cos \theta \delta \theta+l_{p} \cos (\psi+\alpha) F_{\psi} \delta \theta
\end{gathered}
$$

Conservative potentials:

(i)For hood: $\quad \boldsymbol{\delta} \boldsymbol{V}_{\boldsymbol{h}}=\boldsymbol{W} \boldsymbol{\delta} \boldsymbol{y}_{\boldsymbol{h}}$

(ii)For spring: $\quad \delta V_{s}=-k_{c}\left[l_{s}-l_{0 c}\right] \delta l_{s}$

Virtual work:

$$
\begin{aligned}
\delta W & =P_{a} \delta y_{p}-\delta V_{s}-\delta V_{h} \\
& =0
\end{aligned}
$$

Force:

$$
P_{a}=\frac{\delta V_{h}+\delta V_{s}}{\delta y_{p}}
$$

where $\boldsymbol{P}_{\boldsymbol{a}}$ is the vertical force need by a person to open the hood.

\section{Appendix C. Second Link Compression Spring}

Loop closure equations is below:

$$
l_{2} e^{i \phi}+l_{3} e^{i \psi}-l_{4} e^{i \theta}-x_{0}-i y_{0}=0
$$

where angle $\boldsymbol{\theta}$ is the position of the second link.

Velocity coefficients of the system:

$$
\begin{aligned}
&-l_{2} \sin \phi F_{\phi} \delta \theta-l_{3} \sin \psi F_{\psi} \delta \theta+l_{4} \sin \theta \delta \theta=0 \\
&=0 \\
& l_{2} \cos \phi F_{\phi} \delta \theta+l_{3} \cos \psi F_{\psi} \delta \theta-l_{4} \cos \theta \delta \theta=0
\end{aligned}
$$

solved for $\boldsymbol{F}_{\boldsymbol{\psi}}$ and $\boldsymbol{F}_{\boldsymbol{\phi}}$.

Length of springs: 


$$
\begin{aligned}
& l_{s}^{2}=\left(b_{x}-x_{s}\right)^{2}+\left(b_{y}-y_{s}\right)^{2} \\
& b_{x}=b \cos (\theta+\beta) \\
& b_{y}=b \sin (\theta+\beta)
\end{aligned}
$$

Position of center of the hood for $y$ axis:

$$
y_{h}=-y_{0}+l_{2} \sin \phi+a \sin (\psi+\alpha)
$$

Position of the P point for $y$ axis:

$$
y_{p}=-y_{0}+l_{2} \sin \phi+l_{p} \sin (\psi+\alpha)
$$

Virtual displacements:

$$
\begin{aligned}
& \delta l_{s}= \frac{-b\left(b_{x}-x_{s}\right) \sin (\theta+\beta)}{l_{s}} \delta \theta+ \\
& \frac{b\left(b_{y}-y_{s}\right) \cos (\theta+\beta)}{l_{s}} \delta \theta \\
& \delta y_{h}=l_{2} \cos \phi F_{\phi} \delta \theta+a \cos (\psi+\alpha) F_{\psi} \delta \theta \\
& \delta y_{p}=l_{2} \cos \phi F_{\phi} \delta \theta+l_{p} \cos (\psi+\alpha) F_{\psi} \delta \theta
\end{aligned}
$$

Conservative potentials:

(i) For hood: $\quad \boldsymbol{\delta} \boldsymbol{V}_{\boldsymbol{h}}=\boldsymbol{W} \boldsymbol{\delta} \boldsymbol{y}_{\boldsymbol{h}}$

(ii) For spring: $\quad \boldsymbol{\delta} \boldsymbol{V}_{s}=-\boldsymbol{k}_{c}\left[\boldsymbol{l}_{\boldsymbol{s}}-\boldsymbol{l}_{\mathbf{0 c}}\right] \boldsymbol{\delta} \boldsymbol{l}_{\boldsymbol{s}}$

Virtual work:

$$
\begin{aligned}
\boldsymbol{\delta} W & =P_{a} \delta y_{p}-\delta V_{s}-\delta V_{h} \\
& =0
\end{aligned}
$$

Force:

$$
P_{a}=\frac{\delta V_{h}+\delta V_{s}}{\delta y_{p}}
$$

where $\boldsymbol{P}_{\boldsymbol{a}}$ is the vertical force need by a person to open the hood.

\section{Appendix D. First Link Torsion Spring}

Loop closure equations is below:

$$
l_{2} e^{i \theta}+l_{3} e^{i \psi}-e^{i \phi}-x_{0}-i y_{0}=0
$$

where angle $\boldsymbol{\theta}$ is the position of the first link.

Velocity coefficients of the system:

$$
\begin{gathered}
-l_{2} \sin \theta \delta \theta-l_{3} \sin \psi F_{\psi} \delta \theta+l_{4} \sin \phi F_{\phi} \delta \theta \\
=0
\end{gathered}
$$

$l_{2} \cos \theta \delta \theta+l_{3} \cos \psi F_{\psi} \delta \theta-l_{4} \cos \phi F_{\phi} \delta \theta=0$

solved for $\boldsymbol{F}_{\boldsymbol{\psi}}$ and $\boldsymbol{F}_{\boldsymbol{\phi}}$.

Position of center of the hood for $y$ axis:

$$
y_{h}=-y_{0}+l_{2} \sin \theta+a \sin (\psi+\alpha)
$$

Position of the P point for $y$ axis:

$$
y_{p}=-y_{0}+l_{2} \sin \theta+l_{p} \sin (\psi+\alpha)
$$

Virtual displacements:

$$
\begin{aligned}
& \delta y_{h}=l_{2} \cos \theta \delta \theta+a \cos (\psi+\alpha) F_{\psi} \delta \theta \\
& \delta y_{p}=l_{2} \cos \theta \delta \theta+l_{p} \cos (\psi+\alpha) F_{\psi} \delta \theta
\end{aligned}
$$

Conservative potentials:

(i)For hood: $\quad \boldsymbol{\delta} \boldsymbol{V}_{\boldsymbol{h}}=\boldsymbol{W} \boldsymbol{\delta} \boldsymbol{y}_{\boldsymbol{h}}$

(ii) For spring: $\quad \boldsymbol{\delta} \boldsymbol{V}_{s}=\boldsymbol{k}_{t} \boldsymbol{\theta}_{d} \boldsymbol{\delta} \boldsymbol{\theta}+\boldsymbol{k}_{t} \boldsymbol{\theta}_{i d} \boldsymbol{\delta} \boldsymbol{\theta}$

Virtual work:

$$
\delta \boldsymbol{W}=P_{a} \delta y_{p}-\delta V_{s}-\delta V_{h}
$$

Force:

$$
P_{a}=\frac{\delta V_{h}+\delta V_{s}}{\delta y_{p}}
$$

where $\boldsymbol{P}_{\boldsymbol{a}}$ is the vertical force need by a person to open the hood.

\section{Appendix E. Second Link Torsion Spring}

Loop closure equations is below:

$$
l_{2} e^{i \phi}+l_{3} e^{i \psi}-l_{4} e^{i \theta}-x_{0}-i y_{0}=0
$$

where angle $\boldsymbol{\theta}$ is the position of the second link.

Velocity coefficients of the system:

$$
\begin{gathered}
-l_{2} \sin \phi F_{\phi} \delta \theta-l_{3} \sin \psi F_{\psi} \delta \theta+l_{4} \sin \theta \delta \theta \\
=0 \\
l_{2} \cos \phi F_{\phi} \delta \theta+l_{3} \cos \psi F_{\psi} \delta \theta-l_{4} \cos \theta \delta \theta=0
\end{gathered}
$$

solved for $\boldsymbol{F}_{\boldsymbol{\psi}}$ and $\boldsymbol{F}_{\boldsymbol{\phi}}$.

Position of center of the hood for $y$ axis:

$$
y_{h}=-y_{0}+l_{2} \sin \phi+a \sin (\psi+\alpha)
$$

Position of the $\mathrm{P}$ point for $y$ axis:

$$
y_{p}=-y_{0}+l_{2} \sin \phi+l_{p} \sin (\psi+\alpha)
$$

Virtual displacements:

$$
\begin{aligned}
& \delta y_{h}=l_{2} \cos \phi F_{\phi} \delta \theta+a \cos (\psi+\alpha) F_{\psi} \delta \theta \\
& \delta y_{p}=l_{2} \cos \phi F_{\phi} \delta \theta+l_{p} \cos (\psi+\alpha) F_{\psi} \delta \theta
\end{aligned}
$$

Conservative potentials:

$$
\begin{array}{ll}
\text { (i)For hood: } & \boldsymbol{\delta} \boldsymbol{V}_{\boldsymbol{h}}=\boldsymbol{W} \boldsymbol{\delta} \boldsymbol{y}_{\boldsymbol{h}} \\
\text { (ii) For spring: } & \boldsymbol{\delta} \boldsymbol{V}_{\boldsymbol{s}}=\boldsymbol{k}_{\boldsymbol{t}} \boldsymbol{\theta}_{\boldsymbol{d}} \boldsymbol{\delta} \boldsymbol{\theta}+\boldsymbol{k}_{\boldsymbol{t}} \boldsymbol{\theta}_{\boldsymbol{i d}} \boldsymbol{\delta} \boldsymbol{\theta}
\end{array}
$$

Virtual work:

$$
\delta W=P_{a} \delta y_{p}-\delta V_{s}-\delta V_{h}
$$


Force:

$$
P_{a}=\frac{\delta V_{h}+\delta V_{s}}{\delta y_{p}}
$$

where $\boldsymbol{P}_{\boldsymbol{a}}$ is the vertical force need by a person to open the hood. 